\title{
Pronoms impersonnels dans le breton vannetais de Loeiz Herrieu. Syntaxe, sémantique et usages en concurrence avec le passif
}

Impersonal pronouns in Loeiz Herrieu's Vannetais: syntax, semantics and usage in competition with the passive

\section{Mélanie Jouitteau}

\section{OpenEdition Journals}

Édition électronique

URL : https://journals.openedition.org//bl/1196

DOI : $10.4000 / \mathrm{lbl} .1196$

ISSN : 2727-9383

Éditeur

Université de Bretagne Occidentale - UBO

\section{Édition imprimée}

Date de publication : 1 juin 2015

Pagination : 261-280

ISBN : 979-10-92331-16-5

ISSN : $1270-2412$

\section{Référence électronique}

Mélanie Jouitteau, « Pronoms impersonnels dans le breton vannetais de Loeiz Herrieu. Syntaxe, sémantique et usages en concurrence avec le passif », La Bretagne Linguistique [En ligne], 19 | 2015 mis en ligne le 01 mai 2021, consulté le 22 mai 2021. URL : http://journals.openedition.org//bl/1196 DOI : https://doi.org/10.4000/lbl.1196

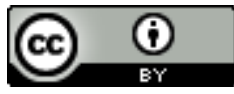

La Bretagne Linguistique est mise à disposition selon les termes de la Licence Creative Commons Attribution 4.0 International. 


\section{Pronoms impersonnels dans le breton vannetais de Loeiz Herrieu. Syntaxe, sémantique et usages en concurrence avec le passif}

$\mathrm{C}$ ette enquête porte sur les pronoms impersonnels dans le breton vannetais de Loeiz Herrieu, tels qu'observés dans le corpus Kammdro an Ankou, journal personnel quotidien en breton vannetais d'un soldat de la guerre de 1914-18 ${ }^{1}$. J'ai choisi ce texte car il est un terrain propice aux occurrences de pronoms impersonnels en $-r$, l'impersonnel d'inflexion verbale propre aux langues celtiques comme illustré en (1)a. La profusion d'impersonnels en $-r$ dans le texte s'explique aisément. D'abord, le corpus est rédigé au temps présent, où la forme en $-r$ est morphologiquement vivante. Ensuite, l'auteur produit de courts récits d'une journée durant quatre ans, pendant lesquels il cumule de multiples raisons tant psychologiques que militaires de procéder à une impersonnalisation répétée des acteurs. La répétition monotone de scénarios semblables offre ce qui se rapproche le plus en corpus de paires minimales pragmatiques : on peut saisir des formes d'impersonnalisations différentes apparaître dans deux scénarios présentés comme rigoureusement identiques.

* CNRS, IKER, UMR 5478, Université de Pau et des Pays de l'Adour, Université Bordeaux III, melanie.jouitteau@iker.cnrs.fr

1. La pagination des références de Kammdro an Ankou données dans cet article est celle de l'édition de 1994 chez Al Liamm. 


$\begin{array}{lll}\text { (1) a. Ne ouier ket } & \text { b. Ne oar ket unan /an den } & \text { c. Ne ouiec'h ket } \\ \text { ne sait.IMP pas } & \text { ne sait pas 1 /le homme } & \text { ne savez pas } \\ \text { «On ne sait pas.» } & & \end{array}$

Dans une première partie, je vais dresser le portrait morphologique et syntaxique des impersonnels dans le corpus. Je comparerai ce portrait aux variations dialectales (autres dialectes du breton), et typologiques (autres langues du monde). Herrieu n'utilise pas la grammaticalisation du cardinal 1 (unan en 1b), mais le pronom an den en (1)b semblable au on français ou au man allemand dans son histoire diachronique de grammaticalisation, si ce n'est qu'il n'est pas restreint à la position de sujet. Les autres formes d'impersonnels sont un pronom vide arbitraire sujet des propositions infinitives (PROarb), les pronoms 2PL (1c) et, la plus utilisée, la forme prototypiquement celtique d'accord verbal en $\mathrm{V}-r$. Je vais montrer que cette forme V-r présente dans le corpus toutes les lectures différentes disponibles aux impersonnels : (i) la lecture générique, de vérité générale (On ne voit bien qu'avec le cœur ${ }^{2}$ ), (ii) la lecture épisodique existentielle (Depuis ce matin, on est en grève à Rennes), qui peut aller jusqu'à une lecture spécifique (On frappe à la porte, on a mangé dans mon assiette), et même (iii) lorsque le locuteur sait spécifiquement de qui il s'agit et choisit de ne pas le préciser (On a demandé à te voir ce matin). Je finis par l'inspection des usages dans les zones laissées optionnelles par la syntaxe : je montre que dans le corpus, les formes en V-r apparaissent dans les mêmes environnements sémantiques, pragmatiques et de discours que le passif. Dans les environnements où la morphologie, la syntaxe et la structure informationnelle autorisent les deux formes, on voit en effet en corpus ces deux formes alterner, et le choix semble être entièrement optionnel.

Le pronom impersonnel est ici considéré comme une entité grammaticale ayant des propriétés précises. Sémantiquement, il est uniquement spécifié par le trait formel sémantique [+humain], ce qui le distingue de l'agent des passifs. En terme de structure informationnelle, un impersonnel est aussi restreint à l'arrière plan. Il ne

2. Sauf mention contraire, les différentes traductions de l'impersonnel de lecture générique viennent des traductions multilingues du Petit Prince de SaintExupéry. http://www.lexilogos.com/textes_multilingues.htm. L'auteur de la traduction a été spécifié lorsque cette information est disponible. 
peut donc être syntaxiquement modifié, focalisé, interrogé, ni mis en tête de relative. Une phrase comme C'est soi-même qu'on admire montre donc que soi-même n'est pas un impersonnel en français. Syntaxiquement, un impersonnel ne peut pas servir de variable liée, c'est-à-dire co-référer avec un quantifieur ${ }^{3}$ : dans $^{\text {Personne }}{ }_{1}$ ne pense qu'on*I/il devrait arrêter, l'impersonnel on n'est possible que s'il ne co-réfère pas avec le sujet personne. Dans les limites de cette étude, je ne traiterai pas des stratégies stylistiques d'impersonnalisation (a), ni des constructions utilisant des pronoms explétifs (b). Je ne traiterai du passif impersonnel (c) que dans la limite où son usage nous éclaire sur l'usage concurrent de pronoms impersonnels.

(1) a. E tachenn La Planche e ta tankirri d'hor c'hemer.

«Des voitures viennent nous prendre à La Planche » p. 107

b. Arc'hoazh ema dle deomp monet kuit ac'henamañ.

« Demain il nous faut partir. »

c. ...da lâret deomp eh eus gwallarru get ur c'heginour...

"...pour nous dire qu'il est arrivé malheur à un cuisinier. »

p. 42

\section{Stratégies absentes en breton: 3SG, 1PL, 3PL}

Une stratégie typologiquement répandue pour créer des impersonnels est l'utilisation du pronom sujet 1PL, par élargissement du contexte épisodique à un contexte maximal humain. Cette stratégie n'est pas représentée chez Herrieu (mais je ne peux pas prouver que parmi les occurrences de pronoms 1PL, aucune ne réfère pour l'auteur maximalement à tout humain).

(2) i véyun bén qu'ac le çheùr.

(3) kan leker gete, lerla nou trouv kler.

(4) We see well only with the heart.

(5) Wi ongl sii gud wid fi-wi haat.

poitevin-saintongeais, Nicolas Martin-Minaret ${ }^{4}$

(6) správně vidíme jen srdcem.

correctement voyons seulement cœur.INSTR tchèque, Milan Rezac, c.p. créole mauricien, Dev Virahsawmy ${ }^{5}$ anglais, Richard Waswo créole jamaïcan, Durrleman-Tame

3. Milan ReZaC et Mélanie JouitTeau, The anatomy of an impersonal pronoun: syntax, semantics and pragmatics of the French impersonal on, manuscrit.

4. http://projetbabel.org/pages/prince.php?p=21, consulté le 06/03/2014.

5. http://kiltir.com/kreol/b0009/dev-virahsawmy-ti-prins-21.shtml, consulté le 06/03/2014. 
Dans les langues comme l'hébreu, le pronom impersonnel le plus fréquent est le pronom 3PL. Cet impersonnel existe en contextes restreints en français (7), mais est absent en breton.

(7) Ils détraquent le temps avec leurs satellites / Ils ont augmenté le tabac.

L'existence d'un pronom impersonnel 3SG comme en finlandais est typologiquement rare, et est clairement absente du breton.

(8) Vain sydämellä näkee hyvin. seulement cœur.ALL voit.3SG bien

finlandais, Torvinen

\section{Stratégies présentes ailleurs en breton : pronom détransitif, cardinal}

Certaines structures se rapprochent du passif par une détransitivisation du verbe, mais restent à la voix active. Dans les langues romanes comme en (9) et (10) et slaves (11), le clitique se peut ainsi avoir un sens impersonnel. En (12), le basque utilise aussi une détransitivisation de ses auxiliaires, et fait un pas de plus vers le passif, avec un accord verbal avec l'objet.

(9) només s'hi veu bé amb el cor.

(10) la s'vé bion mac bou 'l ceur.

catalan, Puig \& Talens

(11) Listky na Lucii se prodávají dobře.

billets.Nom pour Lucie.Acc se vendent bien

"Les billets pour Lucie se vendent bien. » tchèque, Milan Rezac, c.p.

(12) Hezten diren gauzak bakarrik ezagutzen dira, esan zuen azeriak [...] apprivoisé sont-REL chose.D.pA seulement connaissant sont, dit a renard bihotzez besterik ez da ongi ikusten.

cœur.INSTR seulement pas est bien vu

basque

En breton, le proclitique objet réfléchi (en) em n'a pas la lecture impersonnelle. C'est moins clair pour le préfixe nominal réfléchi em-. Kervella ${ }^{6}$ donne emsav, emwerzh avec le sens de 'facile à enlever/ vendre', une lecture compatible avec une lecture impersonnelle. Herrieu, dans le corpus, n'utilise qu'emsav, et uniquement dans le sens religieux de l'Ascension.

6. Frañsez Kervella, Yezhadur bras ar brezhoneg, La Baule, édition Skridoù Breizh, 1947, p. 882. 
Une autre stratégie aisément repérable est la grammaticalisation du cardinal 1 en un pronom impersonnel, comme en anglais ou en castillan. Ce pronom avec une lecture impersonnelle existe en breton (15), mais manifestement pas dans le breton de Herrieu.

(13) One sees well only with the heart.

On voit bien seulement avec le cœur

(14) Sólo se conoce lo que uno domestica - dijo el zorro [...]

seulement se connaît ce que un apprivoise - dit le renard

«On ne connaît bien que ce qu'on apprivoise dit le renard » castillan, Gaston Ringuelet ${ }^{8}$

(15) $\mathrm{Pa}$ ra eun eur fazi, e tleer damanti deon.

quand fait un une faute ${ }^{\circledR}$ doit.IMP expier à.lui

"Quand on fait une faute, il faut en subir les conséquences. » breton (Pleyben), $\mathrm{Ar}$ Gow $^{9}$

Chez Herrieu, le pronom unan est le plus souvent syntaxiquement quantifié ou modifié (pep unan, unan bennak, unan anezhe) sauf dans les fonctions anaphoriques comme dans meur a unan, «plus d'un » (16).

(16) Get an amzer sklasus-mañ e krog ar red-kove meur a unan.

Avec le temps glacial-ci § prend la diarrhée dans plus de un

«Avec ce temps glacial, la diarrhée en prend plus d'un. »

\section{Grammaticalisation de 'humain' : an den}

Dans la plupart des langues romanes et germaniques (aire de Charlemagne), mais aussi en italien et en tchèque, on observe un pronom impersonnel qui est une grammaticalisation souvent transparente du nom lexical pour « humain » $\left(\right.$ on $<$ homme $\left.{ }^{10}\right)$.

7. Sophia A. MALAmUd, « Impersonal indexicals », Journal of Comparative Germanic Linguistics, $\mathrm{n}^{\circ} 14,2011$.

8. https://microtop.ca/lepetitprince/capitulo21.html, consulté le 06/03/2014.

9. Yeun AR Gow, «Les formes impersonnelles en breton : quelques exemples dans le parler de Pleyben ", Les annales de Bretagne ${ }^{\circ}$ 70-4, 1963, p. 497.

10. Andrej Malchukov et Anna Siewierska (dir.), Impersonal Constructions: A cross-linguistic perspective, vol 124, Amsterdam-Philadelphia, Benjamins, 2011. 
(17) On n'vwat bieu qu'aveu l'keûr.

picard d'Ath, R. Huvelle

(18) Man sieht nur mit dem Herzen gut.

on voit seulement avec le cœur bien

(19) kun med hjertet kan man se klart.

allemand, Alvar \& Partzsch

danois, L. \& L. Lisi

seulement avec cœur peut on voir clair

(20) Člověk vidi správně jen srdcem

humain.NOM voit correctement seulement cœurs.INSTR tchèque, M. Rezac

Un impersonnel de ce type est assez répandu dans toute l'aire parlante du breton, à part en dialecte du Léon. Ses formes dialectales varient morphologiquement ${ }^{11}$. On relève :

(21) n'in en breton de Bothoa et en Pélem

an den en trégorrois

un den en trégorrois et à Moëlan

an nen à Tréguier, en Haute-Cornouaille à Quimperlé et à Berrien

den à Plaudren en bord gallo

Un locuteur peut utiliser plusieurs formes (22). La lecture sémantique du pronom impersonnel an den est distincte de la lecture d'espèce sur un syntagme nominal défini (23). En (24), an nen a la distribution d'un pronom, en contraste avec le sujet post-tensé lexical Yann.

(22) Red e vez d 'an den ober e zispign diouz e hounedigez. breton (Trégor), Gros ${ }^{12}$ " II faut régler sa dépense sur son gain. »

Bet e-neus eun den buheziou!

«On a eu de ces vies (difficiles, pénibles, terribles). » breton (Trégor), Gros ${ }^{13}$

(23) Amañ emañ an den ur bleiz evit an den.

ici est le homme un loup pour le homme

«Ici, l'homme est un loup pour l'homme. » breton (Trégor), Gros ${ }^{14}$

(24) Ben 'vez (an nen/eñ) klañv (Yann), ne vez ket gwelet ken. quand est (le homme/lui) malade (Yann) ne est pas vu plus

"Quand on/il/Yann est malade, on/il/Yann ne sort plus.» breton (Rieg), B., 01/2009

11. Mélanie Jouitteau, « Pronom Impersonnel », site wiki ARBRES, 2014, http:// arbres.iker.univ-pau.fr/, consulté le 06/03/2014.

12. Jules GROS, Le trésor du breton parlé III. Le style populaire. Eléments de stylistique trégorroise, Brest, Emgleo Breiz - Brud Nevez, 1984, [première édition 1974], p. 528.

13. Ibidem, p. 180.

14. Ibidem, p. 172. 
L'impersonnel an den chez Herrieu co-réfère aisément avec d'autres impersonnels (PROarb, $-r$ ou 2PL).

(25) Spiañ a raer ${ }_{1}$ ivez linennoù ar Jermaned. Ur gwel kaer en deus an den ${ }_{1}$ ac'hanemañ. épier $₫$ fait.IMP aussi lignes le Allemands. un vue beau $₫$ a le homme de.là «On épie aussi les lignes allemandes. De là, on voit bien. » p. 199

(26) Hañval eo d'an den [ [PRO $\left._{a b b 1}\right]$ bout en un ti gwerzh bras]. pareil est à'le homme être en un maison vente grand "On croirait être dans un grand magasin. »

(27) Fang Breizh a stage ivez doc'h an den , met fang ar vro-mañ ho lonk. fange Bretagne $®$ collait aussi à le homme mais fange le pays-ci vous avale «La boue de Bretagne vous collait aussi, mais celle de ce pays vous avale. » p. 104

Les impersonnels grammaticalisés à partir du nom lexical pour humain sont limités à la position sujet dans les langues romanes ou germaniques. Cette restriction est typologiquement accidentelle. Herrieu utilise an den dans la position de sujet préverbal non-focalisé du vannetais (28), mais aussi comme expérienceur dans un syntagme prépositionnel (29), ou possesseur (30).

(28) Hag ar magoerioù pri-se, an den o zreusahe forzh aes get un taol penn. et le murs terre-là le homme les traverserait fort facile avec un coup tête «Et ces murs de terre, on les traverserait très facilement d'un coup de tête. » p. 114

(29) Forzh aes vehe d'an den fari.

fort aisé serait à le homme se.tromper

"On se tromperait très facilement. »

(30) Skuiziñ a ra daoulagad an den dre forzh sellet doc'hte. fatiguer $₫$ fait deux.œil le homme par force regarder à.eux «On se fatigue à les regarder. »

Le syntagme correspondant pluriel, an dud apparaît aussi avec des lectures impersonnelles prototypiques (31), parfois ambigües avec l'expression militaire « les hommes » (32) mais co-référant avec l'agent d'un passif. Cependant, il est peu plausible qu'un syntagme pluriel ait grammaticalisé en un pronom. On trouve d'ailleurs des occurrences où an dud est modifié (33). 
(31) ...marse talvoudusoc'h eget ne seblant $t_{\text {GEN }}$ d'an dud.

p. 308

« ... peut-être plus important qu'on ne pense. »

(32) ...rak evit paraat na vezvo an dud eh eus kemennet ne vo ket gellet degas gwin ag an davarn.

« ...car pour empêcher qu'on se saoule (que les hommes se saoulent), il est ordonné qu'on ne pourra plus emmener du vin du bar. »

p. 75

(33) Da 3 eur 30 eh on dic'housket get an dud é fichal, é huchal.

« Je suis réveillé à $3 h 30$ par $\approx$ des gens qui bougent, crient. »

p. 76

\section{Dans les infinitives : le pronom silencieux à lecture arbitraire}

Les sujets des infinitives sont phonologiquement nuls dans la plupart des langues d'Europe. La disponibilité d'un pronom vide à lecture arbitraire (noté $\mathrm{PRO}_{a r b}$ ) ne dépend pas d'une propriété de l'infinitive dans laquelle il apparaît, mais des relations de liage anaphorique disponibles. En (34), le sujet de s'impliquer co-réfère obligatoirement avec lui, le syntagme datif de la proposition matrice. En l'absence de ce datif, le sujet de l'infinitive a une interprétation arbitraire.

(34) L'art lui 1 demande de [ $\mathrm{PRO}_{1 / 22}$ s'impliquer totalement]. L'art demande de $\left[\mathrm{PRO}_{\text {arb }}\right.$ s'impliquer totalement].

Chez Herrieu, le pronom vide à référence arbitraire peut coréférer avec un impersonnel en $-r$ (35). En (36), le locuteur observe que dès l'aube, des coups de canon et de fusils éclatent sans but précis. La seule information est que ceux qui tirent, d'un côté ou de l'autre des tranchées, obtiennent une situation où eux-mêmes $\left(_{1}\right)$ empêchent d'autres $\left(_{2}\right)$ de dormir. Des pronoms arbitraires peuvent être référentiellement dépendants, et ne pas co-référer entre eux.

(35) Ar-lerc'h [PRO ${ }_{\text {arb }}$ bout bet mestr], a respontan, e kaver diaes [PRO ${ }_{\text {arb }}$ bout mevel] après $\quad I_{1 M P_{1}}$ être été maître $@$ réponds $@$ trouve. $I M P_{1}$ difficile $I M P_{1}$ être valet « II est dur d'avoir un maître après en avoir été un. »

p. 288

(36) Trouz a raer evit [ $\mathbf{P R O}_{\text {arb }}$ parraat [ $\mathbf{P R O}_{\text {arb }}$ a gousket ]].

bruit $\circledast$ fait.IMP 1 pour $\quad \mathrm{IMP}_{1}$ empêcher $\mathrm{IMP}_{\left.2\right|^{* 1}}$ de dormir

«On fait du bruit pour s'empêcher de dormir. » 


\section{Lecture impersonnelle de seconde personne (= tu, vous)}

L'usage de la seconde personne pour un usage impersonnel est répandu à travers les langues, comme en allemand, en grec (37) ou en anglais (38).

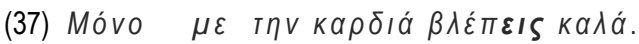

Grec, Takis Kounelis seulement avec le cœur vois.2SG bien

(38) ...you never know.

« Tu ne sais jamais » / « On ne sait jamais. »

Anglais, Malamud ${ }^{15}$

Parmi ces langues, il existe une variation quant à la disponibilité d'une lecture impersonnelle pour les pronoms pluriels. Malamud ${ }^{16}$ note que les pronoms 2PL en anglais non-standard (39) et en allemand (40) sont restreints à une lecture déictique. Ce n'est le cas ni en français (41) ni en breton (42).

(39) $[y / z]$ could come over any time.

"Vous pouvez venir quand vous voulez. » (*On peut venir)

(40) Damals musstet ihr vorsichtig sein.

alors deviez vous prudent être

" En ce temps-là, vous deviez être prudents. » ( ${ }^{*} \ldots$ on devait...)

(41) Avec elle, tu ne sais jamais ! / Avec elle, vous ne savez jamais!

"Avec elle, on ne sait jamais. "

anglais

(Sud-Philadelphie)

(42) Petra e v-malec'h gant ar vilin-se?

«Qu'est-ce qu'on peut moudre avec ce moulin ?» breton (haut-cornouaillais) Evenou ${ }^{17}$

L'usage des formes de tutoiement à travers les dialectes du breton est une question en soi ${ }^{18}$. Cheveau ${ }^{19}$ note par exemple l'absence de formes de tutoiement à Ploemeur, Guidel, Caudan et Quéven. Dans le récit de Herrieu les adresses sont rares, et celles faites à une personne seule, en contexte militaire, sont régulièrement plurielles. L'impersonnel, en tout cas, est clairement 2PL, et tout les paradigmes

15. S. MALAMUd, op. cit.

16. Ibidem.

17. Erwan EvENOU, Description phonologique du breton de Lanvenegen (canton $d u$ Faouët, Cornouaille), thèse Rennes II, 1987, p. 581.

18. M. JouitTEAU, « Tutoiement », ARBRES, op. cit.

19. Loic CheveAu, « Le breton de Ploemeur d'après l'ALBB de P. Le Roux : lecture critique », La Bretagne Linguistique, $\mathrm{n}^{\circ} 15$, Nelly Blanchard et al. (dir.), Brest, CRBC-UBO, 2011, p. 30 : https://journals.openedition.org/lbl/ 
morphologiquement vivants de la personne 2PL peuvent recevoir une lecture impersonnelle. En (43), le pronom 2PL, incorporé dans une préposition ou possessif, co-réfère avec une marque d'impersonnel verbal en $-r$, ou du moins un sous-ensemble de sa référence.

(43) Arvistr a oui a-walc'h ema èl-se a raer ${ }_{2}$ ha mar dahe c'hoant deoc' $h_{2}$ le maîtres ${ }^{\circledR}$ sait assez est comme-ça ${ }^{\circledR}$ fait.IMP et si venait envie à.vous da lakaat an traoù èl m'emaint e vehe klasket c'hwen en $\mathbf{h o}_{2}$ chaochoù. de mettre le choses comme que sont $®$ serait cherché poux dans vos guêtres « Les maîtres savent bien que c'est comme ça qu'on fait, et s'il vous venait à l'esprit d'écrire les choses comme elles sont, on vous chercherait des poux dans la tête. » p. 75

Le pronom 2PL peut aussi être référentiellement autonome : en (44), on voit qu'il est disjoint de l'impersonnel verbal.

(44) Ur penn-kaol dibom a c'houlenner 5 pe 6 blank genoc'h evitoñ. un tête-chou sans.pomme $₫$ demande.IMP cinq ou six blanc avec.vous pour.lui « On demande cinq ou six francs pour un chou non-pommé. »

Le pronom 2PL peut co-référer avec un $\mathrm{PRO}_{a r b}(45)$, ou encore l'agent d'un passif en (46).

(45) Ret eo [PRO arb bout graet ar vicher-se e-pad pevar blez bennak [...]] evit $P R O_{\text {arb }}$ kompren pegen divourrus eo. Arru a rit en ur vourc'h n'anavit ket, lec'h nend eus mui den, na tier lies, [...]. Lojeris hoc'h eus da sevel [...]. E-kreiz an teñvelded ema dav deoc'h klask...

« II faut [PRO arb avoir fait ce métier pendant quatre ans [...]] pour $\mathrm{PRO}_{\text {arb }}$ comprendre à quel point il est désagréable. Vous arrivez dans un bourg que vous ne connaissez pas, désert, souvent détruit et sans maisons [...]. Vous devez vous loger [...]. Dans le noir, vous devez chercher... »

p. 54

(46) Ha tuchant, pand int du-hont, streweter winieg ha ne welit mui nemet o fennoù, et tantôt quand sont côté-là.bas penché dans.le vigne et ne voyez plus que leur têtes e vehe lâret bobelaned gwenn...

§ serait dit papillons blanc

« Et tantôt, quand ils sont là-bas, penchés sur la vigne et qu'on ne voit plus que leurs têtes, on dirait des papillons blancs... »

p. 80

Le contexte prototypique d'apparition des impersonnels 2PL dans le corpus est de loin celui des reprises pronominales filées, qui peuvent s'étendre sur des pages entières. Sémantiquement, et contrairement aux usages habituels de la personne 2PL d'adresse, l'impersonnel 2PL inclut toujours le locuteur. Stylistiquement, 
l'usage de cet impersonnel est même un appel à la projection (« moi, comme vous lecteur ou n'importe qui dans ma situation »).

(47) Amañ e kollahomp c'hoazh amzer neoazh. A pa fall deoc'h monet en un ti, ema ret deoc'h kemer ho tro, en ur steud soudarded arall [...]. A pa vez digoret an nor, ne vez ket lezet nemet tri da vonet en ti àr-un-dro. Hag pand oc'h en ti, ema ret deoc'h gortoz c'hoazh [...]. Neuze e tennit ag ho sac'h roll an traoù a zo lâret deoc'h kemer.

«Mais ici nous perdons encore du temps. Et quand il vous prend d'entrer dans une maison, il vous faut prendre votre tour, dans une file d'autres soldats [...]. Et quand la porte s'ouvre, il n'en est laissé entrer que trois à la fois. Et quand vous êtes dans la maison, il vous faut encore attendre [...]. Alors vous tirez de votre sac les choses qu'il vous a été dit de prendre. "

p. 38

Ces reprises filées seraient impossibles pour l'auteur avec un passif ou une forme en $-r$, par manque de possessif ou de pronom pouvant s'incorporer dans une préposition. L'usage du pronom 2PL n'est cependant pas exclusivement un évitement de la forme en $-r$ défective. On trouve parfois des occurrences isolées, là où un $-r$ aurait pu apparaître : en (48), l'impersonnel d'accord verbal 2PL initie la référence impersonnelle (décrochée de l'impersonnel en $-r$ de la phrase précédente). Elle se trouve sur deux verbes de suite puis disparaît. La phrase suivante utilise un impersonnel en $-r$ référentiellement indépendant. La donnée en (49) vérifie que le verbe irrégulier mont, « aller» en (48) n'est pas défectif pour l'impersonnel.

(48) Kavoù an ti lec'h mah omp é lojiñ en deus 7 lev bennak a hirded, a lârer. A pa yit enne e kerzhit etre div steud stalioù karget a voutailhadoù gwin ag an diaz d'al lein. Oc'hpenn 5 million boutailhad a c'heller lakaat e kavoù Epernay.

« La cave de l'endroit où nous logeons fait, à ce qu'on dit, quelques 7 lieues de long. Et quand vous y allez, vous marchez entre deux rayons de magasin chargés de bouteilles de vin du sol au plafond. On peut loger plus de 5 millions de bouteilles dans les caves d'Epernay. "

(49) da 5 eur 30 eh aer àr-raok.

à 5 h $30 \AA$ va.IMP en-avant

«On avance à 5 h30. »

Le pronom impersonnel 2PL peut se trouver inclus dans un réseau de coréférence déictique. En (50), la coréférence filée sur les trois phrases commence avec un pronom déictique 1PL, poursuit avec un pronom impersonnel $2 \mathrm{PL}$, puis un pronom déictique 1PL. 
En (50), il semble que ce soit l'itérativité de l'action qui rende soudainement l'impersonnel licite.

(50) Monet a raomp a-dastorn-kaer dre ar c'hoad [...]. Dre bep div wezh e tihentit hag eh a ho troad er vouilhenn vlot. Gouloù-red a za da deñvelaat c'hoazh hon hent, goude ma vezont marv...

« Nous avançons à tâtons par le bois, depuis le sentier [...]. De temps en temps, vous dérapez et votre pied termine dans la gadoue. Notre chemin est encore assombri par nos lampes-torches quand elles s'éteignent. »

p. 222

Encore plus surprenant, en (51), l'impersonnel 2PL peut coréférer avec un syntagme 3PL dans la même phrase. Ce décrochage ne se trouve pas avec la forme en $-r$ et est assez inconfortable en français.

(51) Nend eus nemet ar re en deus graet hent èl-se, e-pad ar brezel, e-kreiz an teñvelded mac'hus, sammet gwazh eget loened, àr hentoù goloet a fang, stagus èl toaz bara, c'hwez-brein, edan ar glav yen a santit é redek àr ho kroc'henn toemm, èl barvenn un aotenn, hag a c'hell kompren donded diskoñfort ar c'haezh soudard àr-droad é vonet d'ar bouzellennoù.

" II n'y a que ceux qui ont fait route comme ça, pendant la guerre, dans le noir oppressant, chargés pire que des bêtes, sur des routes recouvertes de gadoue, collante comme de la pâte à pain, puante, sous la pluie froide que vous sentez courir sur votre peau chaude, comme la lame d'un rasoir, qui peuvent comprendre la profondeur de l'inconfort du pauvre fantassin allant aux tranchées. »

p. 99

\section{La forme celtique V-r}

L'impersonnel en $-r$ est syntaxiquement ou bien un pronom sujet incorporé, ou bien la marque de l'accord avec ce pronom. Il est restreint à un trait [+humain] et n'a nulle part de version en pronom fort ou en pronom écho, ce qui est consistant avec sa restriction informationnelle en arrière-plan. L'impersonnel pronominal en $-r$ est syntaxiquement clairement distinct d'un passif ${ }^{20}$. Avec les transitifs, l'agent n'est pas optionnel. L'agent n'apparaît pas dans un groupe prépositionnel. L'objet maintient son marquage, que celui-ci soit un clitique préverbal ou une forme prépositionnelle $a$-.

20. Steve HewitT, « The impersonal in Breton », Journal of Celtic Linguistics 7, 2002, p. 1-39. 
Le pronom impersonnel sujet en $-r$ est un paradigme défectif dans plusieurs dimensions. Les formes $\mathrm{V}-r$ n'existent nulle part avec le verbe kaout, 'avoir' 21 , et elles n'ont pas de forme à l'impératif ${ }^{22}$. $\mathrm{V}-r$ est très vivant au présent, rare au futur et rarissime au passé (52). Les formes en $-r$ sont un paradigme défectif dans tous les dialectes du breton, avec une variation dans cette défectivité.

(52) carte ALBB 353 : «Quand on chante » > formes en $-r$ - un seul passif

carte ALBB 355 : «Quand on chantera » > une demi-douzaine de $-r$ - le reste au passif carte ALBB 354 : «Quand on chantait » > une seule forme en $-r$, le reste 1PL et passif ${ }^{23}$

Je n'ai trouvé chez Herrieu qu'une forme en $-r$ au conditionnel, probablement gelée (ne feher bout karadekoc' $h$, "On ne saurait être plus aimable »). Ces formes morphologiques sont plus vivantes dans d'autres variétés ${ }^{24}$. V-r apparaît avec des verbes transitifs, inergatifs, inaccusatifs - dont le verbe 'être' dont on ne trouve pas chez Herrieu de formes en eh eur, emaer, emeur, emer mais en vezer, en prédication ou sur l'auxiliaire des passifs.

(53)a. E vezer dalc'hmat edan monet àr-raok. (B) est.IMP toujours sous aller en-avant

" On est toujours sur le point d'avancer (partir vers le front). » $\quad$ p. 46

b. ...gwelet e vezer get ar Jermaned...

...vu @ est.IMP par le Allemands

"Nous sommes en vue des Allemands. »

La forme en $-r$ des autres langues celtiques est restreinte à la position sujet. Ceci est un accident typologique ${ }^{25}$. Dans le Nord-

21. Louis LeCLeRC, Grammaire Bretonne du dialecte de Tréguier, Brest, Ar Skol Vrezhoneg, Emgleo Breiz, 1986 [première édition 1906] ; Visant FAVE, Notennou yezadur, Brest, Emgleo Breiz, 1998, [la version en ligne a des corrections] ;

S. Hewitt, op. cit., et F. Kervella, op. cit.

22. F. Kervella, op. cit, p. 190.

23. Pierre Le Roux, Atlas linguistique de la Basse-Bretagne, Paris, HommayChampion, 1927.

24. M. JouitTeau, op. cit.

25. James McCloskeY, «Irish Impersonals in Context », Workshop on Impersonal Pronouns, Paris, CNRS Pouchet, 20 septembre 2011. 
Ouest de la zone parlante, l'impersonnel verbal en $-r$ est, ou a été, généralisé aux prépositions ${ }^{26}$.

(54) Araozor e kerz ar skeud. avant.IMP @ marche le/IMP ombre 'L'ombre (de soi) marche devant soi.'

Fave

(55) Pa zeu tud davedor n' eo ket brao o digouvia re vuan. Quand vient gens vers.IMP ne'est pas beau les dé.convier trop vite «Quand on reçoit du monde, il n'est pas convenable de les congédier trop vite. » Fave

En Léon en breton moderne, les formes en -or des prépositions impersonnelles sont souvent maintenant confondues avec les formes des prépositions impersonnelles de 2PL en $-o c^{\prime} h^{27}$. Dans Herrieu, les formes en $-r$ sont, comme dans les autres langues celtiques, restreintes à la position du sujet incorporé dans la formes verbales (marques d'accord).

$\mathrm{Ci}$-dessous, on voit que la forme $\mathrm{V}-r$ peut lier le syntagme anaphorique an-unan (littéralement /le-un/), et lui donner une lecture impersonnelle. Je n'ai pas trouvé chez Herrieu de forme en an-unan, ni possessive (56), ni réfléchie (57), ni prédicative (58).

(56) Madou an nesa peurliesa a gaver gwelloh evid re an-eun. biens le prochain habituellement ${ }^{\circledR}$ trouve.IMP mieux que ceux le/IMP-un 'On trouve habituellement les biens du prochain préférables aux siens propres.'

(57) Goulenn a reer ouzor an-unan ha n'eo ket an anv-ze eun distresadur [...] demander $₫$ fait.IMP à.IMP le-un si ne'est pas le nom-là un transformation «On se demande si ce nom n'est pas une transformation. »

breton (Cléder), Seite ${ }^{29}$

26. Voir Visant FAVE, « Ar ragano gourfenger or », Feiz ha Breiz, mars-avril 1943, p. 271-272 ; Visant FAVE, "Ar stummoù dibersonel e brezhoneg », Brud Nevez, n93, 1986, p. 69-74; Robert D. Borsley, Janig StePhens, «Agreement and the position of subjects in Breton », Natural Language and Linguistic Theory, $\mathrm{n}^{\circ} 7$, 1989, p. 407-427 ; Visant FAVE, Notennou yezadur, op. cit. ; Visant FAVE, Notes de grammaire bretonne, Mairie de Cléder, 2004, P.-J. Nedelec, Yezadur ar brezoneg, Lesneven, s. n., 1943 ; Y. AR GOW, op. cit ; Pierre TRÉPOS, Grammaire bretonne, Brest, Brud Nevez, 2001 [1968] ; Mélanie JouitTeau et Milan ReZaC, Breton impersonal forms, présentation orale à Celtic Linguistics Conference $\mathrm{n}^{\circ} 7$, Rennes, juin 2012.

27. M. JouitTEAU et M. ReZaC, op. cit., 2012.

28. Y. AR Gow, op. cit.

29. Visant SEITE, O pourmen dre Vreiz-Izel, Levrenn genta, Brest, Emgleo Breiz, 1998, p. 88. 
(58) $\mathrm{Pa}$ gred deor beza an-unan...

quand croit à.IMP être le-un

«Quand on pense être seul... » [Fave]

On peut voir malgré tout dans le corpus que l'impersonnel en $-r$ a assez de traits syntaxiques pour lier un réfléchi (Jouitteau \& Rezac 2012) ${ }^{30}$.

(59) N'em glemmer $_{1}$ ket neoazh ... ne se plaint.IMP pas cependant «On ne se plaint pas cependant. » p. 109 ...lec'h ma c'heller ${ }_{1}$ em 1 astenn ... où que peut.IMP se étendre «... où on peut s'étendre. »

\section{Plasticité référentielle}

La référence calculée pour l'impersonnel varie selon la lecture temporelle et aspectuelle du verbe. Avec une lecture de vérité générale, comme dans un proverbe, les impersonnels sont génériques : ils concernent tout être humains («quel que soit l'humain que l'on considère... ») comme en (61). Plus les phrases deviennent contextualisées et épisodiques, et plus les lectures des impersonnels sujets progressent vers l'existentiel (« il existe un humain tel que... »), ou même le spécifique (« il existe un humain spécifique (qu'éventuellement je connais) tel que...»).

(60) Lecture de vérité générale $>>>$ contextes épisodiques impersonnel générique impersonnel existentiel et spécifique

(61) Ne gaser ket ar chas da jiboes a daolioù bazh!

«On n'envoie pas les chiens chasser à coups de bâtons!» proverbe, p. 98

(62) Marse e vehe gwell bout dispartiet-groñs doc'h ar re a garer?

«Peut-être vaudrait-il mieux être séparé complètement de ceux qu'on aime? » p. 60

En (63), les contextes sont de vérité générale à l'intérieur d'un espace de temps défini, visibilisé par une quantification adverbiale. L'impersonnel réfère à tous les humains à l'intérieur de ce contexte restreint.

30. M. Jouitteau et M. ReZaC, op. cit. 
(63) a. Tud da labourat douar ne gaver ket mui. gens pour travailler terre ne trouve.IMP pas plus "On ne trouve plus de gens pour travailler la terre. »

b. rakèl-se 'vez bepred a pa araoker en un tachad bennak. car comme-ça 'est toujours et quand avance.IMP dans un endroit quelconque « Car il en est toujours ainsi quand on opère une avancée. »

c. Bremañ e kaver Breizhiz e kement lec'h a zo. maintenant $@$ trouve bretons dans autant lieu $@$ y.a « Maintenant, on trouve des bretons partout. »

C'est cette même lecture qu'on obtient avec les verbes de perception en (64), ce qui est perçu est très contextualisé et ponctuel, mais n'importe quel humain le percevrait.

(64) a. ...hag emberr e klever lod é tiroc'hal « ... et bientôt on en entend certain ronfler. »

b. Ar lein an douar e weler eskern tud... "Sur le sol, on voit des os humain. »

Les actions ponctuelles très répétées continuent de quantifier sur un ensemble humain important, mais l'épisodicité du procès commence à donner à l'impersonnel une lecture s'approchant du déictique. Ces impersonnels se différencient d'un pronom 1PL par la possibilité d'exceptions individuelles à une règle générale. C'est cette lecture qu'on obtient typiquement pour les rumeurs ou les nommages :

(65) ... en un toull, leh mah aer da staotet, ...

" dans un trou où on va pisser »

(66) Evit diskuizh pevar devezh, a lârer. " pour se reposer quatre jours, à ce qu'on dit. » ur gêr àr ar maez a raer La Cendrière anezhi. un lieu en campagne qu'on appelle La Cendrière.

p. 36

Les actions ponctuelles répétées peu de fois tirent encore un peu plus vers des sujets impersonnels individués (68), jusqu'aux actions ponctuelles uniques qui ne réfèrent plus qu'à un seul individu, avec une lecture clairement existentielle spécifique (69).

(68) a. ...toulloù goulle a ya àr strishaat seul mui ma tostaer d' o fozelloù. p. 46 trous vide $₫$ va en rétrécir à.mesure plus que approche.IMP de leur tranchées « Des trous vides qui vont rétrécissant à mesure qu'on s'approche de leurs tranchées. » 
b. Ne denner àr netra, nag àr hani.

"On ne tire sur rien, ni sur personne. »

(69) a. Kaer em eus skoiñ àr zor an ti a zo merket din, nen daer ket da zigor. p. 19

" J'ai beau frapper à la porte de la maison qui m'est indiquée, on ne vient pas ouvrir. »

b. Henoazh e oan kousket dous àr ma flouz a pa zaer da lâret din... p. 124 « Mais j'étais profondément endormi sur ma paille quand on est venu me dire... »

Le référent peut même être cité nommément, et on obtient alors une stratégie pragmatique de préservation de la face (70), raison probable du grand nombre d'usages de l'impersonnel pour référer de façon vague à la hiérarchie militaire (71).

(70) Kemenniñ a ra an adjudant dezhoñ deval e zaoulagad: an arall ne ra van. ordonner $₫$ fait le adjudant à.lui baisser son deux.oeil: le autre ne fait pas Hag e c'hrataer dezhoñ daou zeiz muioc'h ag al labour spletus ema ec'h ober ! et $®$ donne à.lui deux jour plus de le travail utile est à faire «L'adjudant lui ordonne de baisser les yeux : l'autre n'en fait rien. Et on le gratifie de deux jours supplémentaires de son utile travail ! »

(71) Hor sevel a raer ac'hanemañ èlkent!

nous lève $₫$ fait.IMP de.ici tout.de.même

«On nous enlève tout de même d'ici! »

La plasticité de référence a une autre dimension : l'impersonnel peut inclure le locuteur (72), mais aussi l'exclure, seul (73), ou encore accompagné (71).

(72) Un noz eh araoker pemp paz; an deiz àr-lec'h e kiler pemp arall...

«Une nuit on avance de cinq pas, le jour suivant on recule de cinq autres. » $\quad$ p. 85

(73) Ma c'has a raer d'Epernay en-dro...

«On m'envoie de nouveau à Epernay. »

La plasticité de l'impersonnel en - $r$ n'a pas pu être entièrement testée en corpus. Les possibilités restantes sont l'adresse et l'autoréférence (-On a pris ses cachets, madame X? - Oh non, jamais en public, on a sa fierté!).

\section{Quelle différence sémantique avec le 1PL?}

Des IMP en $-r$ ont parfois clairement une lecture 1PL, comme en (74) et (75). C'est le résultat d'une conjonction accidentelle de deux facteurs : (i) un élément temporel d'épisodicité comme les 
adverbes goude, «après » ou ar wech-mañ, «cette fois-ci »; et (ii) une inclusion du locuteur dans le groupe sujet.

(74) ha goude e kaner get muioc'h a volontez vat eget a emglev. et après $₫$ chante.IMP avec plus de volonté bonne que de entente "Et on chante après avec plus de bonne volonté que d'écoute. »

(75) Ar wech-mañ, e weler GEN/PL peseurt labour a c'heller ${ }_{G E N}$ ober a pa vez emglev etre kanonerion ha soudarded àr droad. An hanter bihanoc'h a dud a goller ${ }_{1 p L}$ hag an hanter muioc' $h$ a hent a raer ${ }_{1 P L}$.

" Cette fois-ci, on GEN/PL voit quel travail on $_{\text {GEN/PLL }}$ peut faire quand il y a entente entre canonniers et fantassins. $\mathbf{O n}_{1 \mathrm{PL}}$ perd moitié moins d'hommes, et on $\mathbf{n}_{1 \mathrm{PL}}$ fait moitié plus de chemin. »

p. 138

\section{Les pronoms impersonnels et 1PL peuvent co-référer.}

(76) a. Akoursat a raomp doc'h hon toulloù nevez hag o c'hempenn a raer a-nebeudigoù. « Nous nous habituons à nos nouveaux trous et on les nettoie petit à petit. » p. 214

b. Lakaar a raer dour àr an tan hag un hantereur àr-lerc'h e c'hellomp reiñ kafe d'ar gompagnunezh...

« On met de l'eau sur le feu et une demi-heure plus tard nous pouvons donner du café à la compagnie. »

p. 51

Il est plausible que, comme l'impersonnel français, la forme en $-r$ s'arrange mieux des exceptions individuelles à une généralité exprimée qu'un pronom 1PL. En (77), Herrieu s'inclut d'abord dans le groupe qui prend part à la discussion sur ceux qui s'expriment mal en français (il est par ailleurs fier de sa propre maîtrise du français), puis il se distance du groupe qui se moque des bretons par l'usage d'un impersonnel en $-r$.

(77) Savet a zo kaoz genomp ag ar re a gomz fall c'halleg, hag e raer goap ag ar Vretoned [...] èl rezon.

« II nous vient d'évoquer ceux qui parlent mal français, et on se moque, bien entendu, des bretons. »

p. 42

\section{Quelle différence d'emploi avec le passif?}

L'agent du passif et une forme en $-r$ sont sémantiquement compatibles et peuvent co-référer.

(78) ... un tammig douar labour lec'h ma vez hadet gwinizh, jenofl pe betrav-sukr, hag a arganter evit beviñ an tiegezh. 
« Un bout de terre où on sème [sont semés] du blé, des œillets ou de la betterave à sucre, qu'on vend pour faire vivre le foyer. »

Le passif peut évidemment suppléer à la défectivité de l'impersonnel : le temps passé et futur, le conditionnel, ou les agents non-humains. L'auxiliaire du passif peut aussi marquer l'habitude (vez), alors que V-r n'est compatible avec une telle morphologie que si l'impersonnel est le sujet patient d'un passif comme en $(53) \mathrm{b}^{31}$. Qu'est-ce qui préside alors au choix entre les deux lorsqu'ils sont également autorisés syntaxiquement ? Dans les exemples ci-dessous, je montre que dans toutes les lectures attachées aux environnements prototypiques que nous venons de voir pour l'impersonnel $-r$, un passif est aussi présent en corpus. L'agent du passif, avec un auxiliaire habituatif (vez), a des lectures génériques (79), et est utilisé pour les verbes de perception et de nommage (80). Il devient plus existentiel dès les contextes épisodiques itératifs (81), et on le trouve aussi dans un contexte clairement épisodique (82).

(79) Lâret e vez atav : An nep a ra goap, Doc'he revre stag. dit $\circledast$ est.HAB aussi : le qui $\circledast$ fait blague à son cul $\circledast$ colle "On dit aussi : celui qui se moque, ça le suit»

Tammigoù kenwerzh a vez graetivez.

Morceaux.petits commerce $₫$ est.HAB fait aussi

"On fait aussi un peu de commerce. »

(80) a. Ne vez ket gwelet kalz a ilizoù gotek kaeroc'h. ne est.HAB pas vu beaucoup de églises gothiques beau.plus p. 81 «On ne voit pas beaucoup d'églises gothiques plus belles. »

b. un dachenn paour ha diamen a vez graet Araja anezhi. un champ ${ }_{1}$ pauvre et isolé @est.HAB fait Araja de elle ${ }_{1}$ "On appelle Araja un champ pauvre et isolé. »

(81) Sellet omp èl re kar ha boket a raer deomp kent hon ambroug d'hor gwele.

regardé sommes comme ceux aimé et baiser $₫$ fait.IMP à.nous jusqu'à nous $V$ à.notre lit

«On nous regarde comme de proches et on nous embrasse jusqu'à nous accompagner à notre lit. »

(82) Kavet a zo doc'htu ur moranv dezhoñ: "Fil-de-fer"! trouvé $®$ est de.suite un surnom à.lui : Fil-de-fer «On lui trouve tout de suite un surnom : Fil-de-fer. »

31. Par ailleurs, le passif est plus utilisé en breton qu'en français, entre autres car il est utilisable avec des intransitifs : E-pad o labour eh eus tennet àr ma zud, littéralement «Il est été tiré sur mes hommes pendant leur travail. » p. 180. 


\section{Conclusion}

L'expression de l'impersonnel chez Herrieu est contrainte par deux facteurs : l'inventaire global des pronoms impersonnels disponibles dans son dialecte et la défectivité de chacune des stratégies disponibles. Herrieu n'utilise pas l'impersonnel unan présent dans d'autres dialectes. Il utilise peu mais régulièrement le pronom an den, et dans des fonctions non-sujet agrammaticales en français, la langue majoritaire dans laquelle il vit au front.

\begin{tabular}{|l|l|c|c|c|}
\hline Pronom & Typologiquement & Breton & Herrieu & Français \\
\hline 3SG & $\sqrt{\text { finlandais }}$ & - & - & - \\
\hline 1PL & $\sqrt{ }$ anglais, tchèque... & - & - & - \\
\hline 3PL & $\sqrt{\text { hébreu }}$ & - & - & restreint \\
\hline réfléchi & $\sqrt{ }$ langues romanes, tchèque... & (préfixe em-) & - & restreint \\
\hline cardinal 1, one & $\sqrt{ }$ anglais, castillan & $\sqrt{ }$ & - & - \\
\hline 2SG & $\sqrt{ }$ anglais, français, allemand & $\sqrt{ }$ & - & $\sqrt{ }$ \\
\hline 2PL & $\sqrt{\text { français }}$ & $\sqrt{ }$ & $\sqrt{ }$ & $\sqrt{ }$ \\
\hline PROarb & $\sqrt{ }$ très répandu & $\sqrt{ }$ & $\sqrt{ }$ \\
\hline Man, on, den & $\sqrt{ }$ Europe de l'Ouest/Sud & $\sqrt{ }$ & $\sqrt{ }$ & $\sqrt{ }$ \\
\hline formes type $-r$ & $\sqrt{ }$ celtique & $\sqrt{ }$ & $\sqrt{ }$ & - \\
\hline
\end{tabular}

Les facteurs syntaxiques ou sémantiques ne semblent dicter les usages des différents impersonnels que par retranchement, en interdisant certains accès. Seul le pronom vide $\mathrm{PRO}$ à référence arbitraire a syntaxiquement accès aux infinitives, où il n'a pas de concurrence. Dans les propositions tensées, quatre stratégies s'affrontent: an den, $-r$, les pronoms 2PL et le passif. Herrieu utilise massivement les formes celtiques en $-r$, et on a vu que la plasticité référentielle de cette forme remplit tout le cahier des charges d'un impersonnel. Le paradigme en $-r$ breton est cependant défectif, et encore plus chez Herrieu qui n'utilise pas les formes prépositionnelles en $-r$ du Nord-Ouest. Il n'a probablement plus de productivité non plus dans le paradigme du conditionnel. Cette forme est donc suppléée par les autres. Le passif peut toujours être choisi là où une forme en $-r$ serait licite. Les pronoms 2PL ne sont pas défectifs, mais ne sont utilisés que par à coups, pour des métaphores filées, et probablement pour construire stylistiquement une proximité avec le lecteur. 